\title{
Epilepsy in pregnancy
}

\author{
Hordagoda $\mathbf{H}^{1}$
}

E pilepsy is the most common neurologic condition in pregnancy. Therefore all doctors involved should know how to counsel women with epilepsy as they are considering pregnancy. Over 90 percent of women with epilepsy have a normal pregnancy ${ }^{1}$. This should be emphasized to the patient who is likely to have many fears and anxieties regarding the risks. The management of pregnancy and epilepsy will be discussed here.

\section{Preconception management}

Preconception counseling is important for all women of child bearing years because many pregnancies are unplanned and the risks of complications can be minimized by interventions before and early in pregnancy ${ }^{2}$.

\section{Contraception}

Women with epilepsy should be aware that hormonal contraceptive failure may occur with antiepileptic drugs (AEDs) which are inducers of the hepatic cytochrome P-450 system (phenytoin, phenobarbital, carbamazepine, oxcarbazepine, primidone, topiramate).

\section{Folic acid supplementation}

Low serum folate levels in women with epilepsy are independently associated with an increased risk of major fetal malformations ${ }^{3}$. It has not yet been conclusively determined if folic acid supplementation prevents neural tube defects in women receiving AEDs. An observational study in women with epilepsy found that the incidence of neural tube defects were

${ }^{1}$ Consultant Neurologist, District General Hospital, Ampara, Sri Lanka.

Correspondence: Hemal Hordagoda

E-mail: hhordagoda@gmail.com

Competing interests: None similar in women who reported preconceptual use of folic acid compared to those who reported to have begun folic acid supplementation later in pregnancy ${ }^{4}$. The 2009 American Academy of Neurology and American Epilepsy Society guidelines state that data are insufficient to determine whether doses higher than $0.4 \mathrm{mg}$ offer greater protective benefits ${ }^{5}$. The American College of Obstetricians and Gynecologists recommend $4.0 \mathrm{mg}$ of folic acid daily for women at risk of having an offspring with neural tube defects (including women taking AEDs) ${ }^{6}$.

\section{Choice of antiepileptic drug}

If it is felt that medications cannot be withdrawn, the patient should take the most suitable medication for the seizure type. The optimal treatment of women with epilepsy who are of childbearing age is unclear. As it would be problematic to perform randomized controlled trials assigning women with epilepsy to various anticonvulsant choices prior to pregnancy, most of the available data regarding outcome of pregnancy on anticonvulsants come from matched cohort observational studies such as pregnancy registries.

Since there is no agreement as to which AED is most or least teratogenic, the AED that stops seizures in a given patient is the one that should be used. An exception is valproate. Early results from pregnancy registries and most recent cohort studies suggest a trend toward higher teratogenicity with valproate than with other AEDs ${ }^{7}$. It is reasonable to avoid valproate in women planning to become pregnant whose seizures can be adequately controlled with other AEDs. If valproate is used, high plasma levels $(>70 \mu \mathrm{g} / \mathrm{mL})$ should be avoided unless necessary to control seizures, and the drug should be given in three/four divided doses daily ${ }^{9}$.
Other recommendations:

- The AED should be administered at the lowest dose and lowest plasma level that protects against seizures.

- The plasma drug level should be monitored regularly during pregnancy including, if available, the physiologically important free or unbound drug concentration.

- Polypharmacy should be avoided where possible.

- In established pregnancy, decisions to alternate AED therapy should not be undertaken solely to reduce teratogenic risk, but for other reasons ${ }^{1}$. Changing AEDs may precipitate seizures. Overlapping AEDs during the change exposes the fetus to effects of an additional AED and polypharmacy. There is limited advantage in changing AEDs if pregnancy has already been established.

\section{Management in pregnancy and delivery}

While all the usual recommendations for women of child bearing potential apply, including preconceptual and ongoing use of folic acid, calcium and Vitamin D, additional consideration must be given to the need for adjustment of anticonvulsant therapy. Many women who experience increased seizure frequency during pregnancy are sleep-deprived or noncompliant because of concerns about the effects of the medication on the developing fetus. Preconception counseling may play a role in minimizing this risk ${ }^{2}$. It is important to emphasize the importance of adequate sleep, medical compliance, and minimizing stress and other factors known to precipitate seizures.

\section{Screening for malformations}

Screening for major anomalies pro- 
vides the patient with the opportunity to terminate the pregnancy if a malformation is present. Even if the patient would not consider termination, it may be helpful to know if a major defect is present in order to plan the optimum mode of delivery and to refer the patient to a center with specialty pediatric services for delivery.

\section{Drug levels and dose adjustment}

Pregnancy is accompanied by many alterations in drug metabolism, including increased liver metabolism, renal clearance, and volume of distribution, and decreased gastrointestinal absorption and plasma protein binding $^{8}$. For example, for AEDs that are highly protein bound such as phenytoin, valproate, the total plasma drug level may decrease with impaired protein binding, but the physiologically important free or unbound drug concentration may not change. As a result, free drug levels for these AEDs may be more reliable during pregnancy. However, medication dosage should be adjusted if the patient's seizures are not controlled, not relying solely on the free or total level that has decreased.

\section{Vitamin K supplementation}

The 2009 AAN AES guidelines concluded that there was not sufficient evidence to recommend for or against this practice $^{5}$. Most physicians recommend administration of prophylactic vitamin K (10 to $20 \mathrm{mg} /$ day) during the last month of pregnancy to women treated with AEDs to protect the child against severe postnatal bleeding due to a deficiency in vitamin K-dependent clotting factors ${ }^{5}$.

\section{At delivery}

Most women have a normal vaginal delivery ${ }^{10}$. However, elective cesarean section may be justified in women with frequent seizures during the third trimester or a history of status epilepticus during severe stress ${ }^{11}$. Doses must not be missed during the period of labor. Convulsive seizures during labor and delivery should be treated promptly with intravenous benzodiazepines; lorazepam is considered the drug of choice ${ }^{10}$. Magnesium sulfate is not an appropriate alternative for epileptic seizures. However, when seizures first present during the third trimester of pregnancy or the early postpartum period, it may be difficult to distinguish eclampsia from a new onset or late relapse of epilepsy. In these cases, treatment of eclampsia and evaluation of other etiologies for the seizure is warranted.

\section{Management in the postpartum period}

If the AED dose has been altered during pregnancy, a return to prepregnancy levels should be considered during the first few weeks after delivery. The mother needs to be advised of the importance of adequate rest, sleep and compliance with drug therapy. Precautions need to be taken to protect the infant if the mother has a seizure. It is prudent, for example, to have another person present when the mother bathes the child. In addition, the baby should be changed on the floor or an alternative safe position.

\section{Breast feeding}

There is no evidence to determine whether this form of AED exposure has clinical effects on the newborn ${ }^{5}$. Most experts believe that taking AEDs does not generally contraindicate breast feeding, as probable benefits outweigh risks ${ }^{1}$.

Problems tend to occur only with the sedative drugs, such as phenobarbital, primidone, or benzodiazepines. Exposure to these drugs may cause the child to become irritable, fall asleep shortly after beginning to nurse, or fail to thrive. If this occurs, breast feeding may need to be discontinued but can be retried one week later.

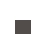

\section{REFERENCES}

1. Practice parameter: management issues for women with epilepsy (summary statement). Report of the Quality Standards Subcommittee of the American Academy of Neurology. Neurology 1998; 51: 944.

2. Sabers A. Influences on seizure activity in pregnant women with epilepsy. Epilepsy Behav 2009; 15: 230.

3. Kaaja E, Kaaja R, Hiilesmaa V. Major malformations in offspring of women with epilepsy. Neurology 2003; 60: 575.

4. Morrow JI, Hunt SJ, Russell AJ, et al. Folic acid use and major congenital malformations in offspring of women with epilepsy: a prospective study from the UK Epilepsy and Pregnancy Register. J Neurol Neurosurg Psychiatry 2009; 80: 506.

5. Harden CL, Pennell PB, Koppel BS, et al. Practice parameter update: management issues for women with epilepsy-focus on pregnancy (an evidence-based review): vitamin $\mathrm{K}$, folic acid, blood levels, and breastfeeding: report of the Quality Standards Subcommittee and Therapeutics and Technology Assessment Subcommittee of the American Academy of Neurology and American Epilepsy Society. Neurology 2009; 73: 142.

6. http://guideline.gov/summary/summary.aspx?doc_id=3994\&nbr=3131.

7. Tomson T, Perucca E, Battino D. Navigating toward fetal and maternal health: the challenge of treating epilepsy in pregnancy. Epilepsia 2004; 45: 1171.

8. Nau, H, Schmidt-Gollwitzer, M, Kuhnz, W, et al. Antiepileptic drug disposition, protein binding and estradiol/progesterone serum concentration ratios during pregnancy. In: Advances in epileptology: XVth International Symposium. Porter, R, Mattson, R, Ward, AA Jr, et al (Eds), Raven, New York, 1984. p.239.

9. Samrén EB, van Duijn CM, Koch S, et al. Maternal use of antiepileptic drugs and the risk of major congenital malformations: a joint European prospective study of human teratogenesis associated with maternal epilepsy. Epilepsia 1997; 38: 981.

10. Yerby MS. Problems and management of the pregnant woman with epilepsy. Epilepsia 1987; 28 Suppl 3: S29.

11. Knight $A H$, Rhind EG. Epilepsy and pregnancy: a study of 153 pregnancies in 59 patients. Epilepsia 1975; 16: 99. 